\title{
On the structure and the qualitative behavior of an economic model
}

Hamdy A El-Metwally*

"Correspondence:

helmetwally2001@yahoo.com

Department of Mathematics, Rabigh College of Science and Arts,

King Abdulaziz University, P.O. Box

344, Rabigh, 21911, Saudi Arabia

Permanent address: Department of

Mathematics, Faculty of Science,

Mansoura University, Mansoura,

35516, Egypt

\begin{abstract}
In this paper, we build an economic model of a non-linear system of difference equations and present a qualitative study for the obtained model, where a mathematical model of a bounded rationality multiple game with an exponential demand function will be introduced, and then we obtain the equilibrium points of the model and classify if they are locally stable or not. Also, we investigate the boundedness and global convergence of solutions for the obtained system.
\end{abstract}

Keywords: difference equations; economic model; boundedness; global stability

\section{Introduction}

In the recent years, the study of the bounded rationality duopoly game has attracted a very high attention. In 1998 Bischi and Naimzada [1] introduced the bounded rationality duopoly game as a modification of the original model work of Cournot [2], where they proposed the duopoly game which describes a market with two players producing homogeneous goods, updating their production strategies in order to maximize their profits. Each player thinks with bounded rationality, adjusts his output according to the expected marginal profit, therefore the decision of each player depends on local information about his output. Also, they have studied the bounded rationality duopoly game with a simple case when the demand function and the cost function are linear [1]. Recently, many works of bounded rationality duopoly game have been studied [1,3-11]. Agiza et al. [5] studied the complex dynamics in a bounded rationality duopoly game with a nonlinear demand function and a linear cost function. The asymptotic behavior of the economic model was investigated by El-Metwally [12].

The main aim for this paper is to analyze the dynamics of a nonlinear discrete-time map generated by a bounded rationality duopoly game with an exponential demand function. In Section 2 we present and describe a bounded rationality duopoly game with an exponential demand function. The existence of the equilibrium points of the obtained model and the studying of their local stability are given in Section 3. The boundedness of the solutions is studied in Section 4. Finally, Section 5 is concerned with the global attractivity of the solutions for the obtained system.

Now consider the following first-order system of difference equations:

$$
\left.\begin{array}{l}
x_{n+1}=f\left(x_{n}, y_{n}\right), \\
y_{n+1}=g\left(x_{n}, y_{n}\right),
\end{array}\right\} \quad n=0,1,2, \ldots
$$

\section{Springer}

where $f$ and $g$ are continuous functions on a subset $S \subset R^{2}$.

O 2013 El-Metwally; licensee Springer. This is an Open Access article distributed under the terms of the Creative Commons Attribution License (http://creativecommons.org/licenses/by/2.0), which permits unrestricted use, distribution, and reproduction in any medium, provided the original work is properly cited. 
Definition System $(*)$ is competitive if $f(x ; y)$ is non-decreasing in $x$ and non-increasing in $y$, and $g(x ; y)$ is non-increasing in $x$ and non-decreasing in $y$. If both $f$ and $g$ are nondecreasing in $x$ and $y$, System $(*)$ is cooperative. Competitive and cooperative maps are defined similarly. Strongly competitive systems of difference equations or strongly competitive maps are those for which the functions $f$ and $g$ are coordinate-wise strictly monotone.

Theorem A [13] Let $T=(f, g)$ be a monotone map on a closed and bounded rectangular region $S \subset R^{2}$. Suppose that $T$ has a unique fixed point $E=(\bar{x}, \bar{y})$ in $S$. Then $E$ is a global attractor of $T$ on $S$.

\section{The model}

We consider a Cournot duopoly game with $q_{i}$ denoting the quantity supplied by firm $i=$ 1 , 2. In addition, let $P\left(q_{i}+q_{j}\right), i \neq j$, denote a twice differentiable and non-increasing inverse demand function and let $C_{i}\left(q_{i}\right)$ denote the twice differentiable increasing cost function. For the firm $i$, the profit resulting from the above Cournot game is given by

$$
\Pi_{i}=P\left(q_{i}+q_{j}\right) q_{i}-C_{i}\left(q_{i}\right) .
$$

Since the information in the oligopoly market is incomplete, the bounded rational players have no complete knowledge of the market, hence they make their output decisions on a local estimate of the expected marginal profit $\frac{\partial \Pi_{i}}{\partial q_{i}}$ [14]. If the marginal profit is positive (negative), it increases (decreases) its production $q_{i}$ at the next period output. Therefore the dynamical equation of the bounded rationality player $i$ has the form

$$
q_{i}(t+1)=q_{i}(t)+v_{i} q_{i}(t) \frac{\partial \Pi_{i}}{\partial q_{i}(t)}, \quad i=1,2,
$$

where $v_{i}$ is a positive parameter which represents the relative speed of adjustment. Bischi and Naimazada studied the dynamical behavior of the bounded duopoly game with a linear demand function [14].

To make the bounded rationality duopoly game more realistic, we assume that the demand function $f(Q)$ has the exponential form (see [15])

$$
f(Q)=a e^{-Q}=a e^{-\left(q_{1}+q_{2}\right)},
$$

where $a$ is a parameter of maximum price in the market. The exponential demand function has the good properties of non-zero or non-negative prices and finite prices when the total quantity in the market $Q$ tends to zero. So, we think that the exponential demand function is a good alternative to the linear demand function and makes the game more realistic. Also, we consider the cost function is linear and is given by

$$
c_{i}\left(q_{i}\right)=c_{i} q_{i}, \quad i=1,2,
$$

where $c_{i}$ is the marginal cost of the $i$ th firm. Thus the profit of the $i$ th firm is given by

$$
\Pi_{i}\left(q_{1}, q_{2}\right)=a q_{i} e^{-\left(q_{1}+q_{2}\right)}-c_{i} q_{i} .
$$


Then marginal profit of $i$ th firm is

$$
\frac{\partial \Pi_{i}}{\partial q_{i}(t)}=a\left(1-q_{i}\right) e^{-\left(q_{1}+q_{2}\right)}-c_{i}, \quad i=1,2 .
$$

Thus the repeated duopoly game of bounded rationality by using Eq. (2) is given by

$$
q_{i}(t+1)=q_{i}(t)+v_{i} q_{i}\left(a\left(1-q_{i}\right) e^{-\left(q_{1}+q_{2}\right)}-c_{i}\right) .
$$

Therefore the discrete two-dimensional map of the game has the form

$$
T:\left\{\begin{array}{l}
q_{1}(t+1)=q_{1}(t)+v_{1} q_{1}\left(a\left(1-q_{1}\right) e^{-\left(q_{1}+q_{2}\right)}-c_{1}\right) \\
q_{2}(t+1)=q_{2}(t)+v_{2} q_{2}\left(a\left(1-q_{2}\right) e^{-\left(q_{1}+q_{2}\right)}-c_{2}\right)
\end{array}\right.
$$

Now we can rewrite this system in the following form:

$$
\left\{\begin{array}{l}
x_{n+1}=\left(1-\alpha_{1}\right) x_{n}+\beta_{1}\left(1-x_{n}\right) x_{n} e^{-\left(x_{n}+y_{n}\right)}, \\
y_{n+1}=\left(1-\alpha_{2}\right) y_{n}+\beta_{2}\left(1-y_{n}\right) y_{n} e^{-\left(x_{n}+y_{n}\right)}
\end{array}\right.
$$

where $x_{n}=q_{1}(t), y_{n}=q_{2}(t), \alpha_{i}=v_{i} c_{i} \in(0, \infty)$, and $\beta_{i}=v_{i} a \in(0, \infty), i=1,2$.

\section{Local stability of the equilibrium points}

In this section, we examine the existence of non-negative equilibrium points of System (9) and then give a powerful criterion for the asymptotic stability of the obtained points.

Proposition 1 (1) When $\alpha_{1} \geq \beta_{1}$ and $\alpha_{2} \geq \beta_{2}$, System (9) has a unique equilibrium point $E_{0}=(0,0)$.

(2) When $\alpha_{1}<\beta_{1}$ and $\alpha_{2}<\beta_{2}$, System (9) has two equilibrium points $E_{1}=\left(x^{*}, 0\right)$ and $E_{2}=\left(0, y^{*}\right)$, where $x^{*}$ and $y^{*}$ satisfy $\alpha_{1}=\beta_{1}\left(1-x^{*}\right) e^{-x^{*}}$ and $\alpha_{2}=\beta_{2}\left(1-y^{*}\right) e^{-y^{*}}$, respectively.

(3) When $\alpha_{1}<\beta_{1} e^{\frac{\alpha_{2} \beta_{1}}{\alpha_{1}}-1}$, System (9) has a unique positive equilibrium point $E_{3}=\left(u^{*}, v^{*}\right)$, where $u^{*}$ satisfies $\alpha_{1}=\rho_{1}\left(1-u^{*}\right) e^{-\gamma_{1} u^{*}}, v^{*}=\frac{\alpha_{2} \beta_{1}}{\alpha_{1} \beta_{2}} u^{*}-\frac{\alpha_{2} \beta_{1}}{\alpha_{1} \beta_{2}}+1, \gamma_{1}=1+\frac{\alpha_{2} \beta_{1}}{\alpha_{1} \beta_{2}}$ and $\rho_{1}=\beta_{1} e^{\gamma_{1}-2}$.

Proof Observe that the equilibrium points of System (9) are given by the relations

$$
\bar{x}=\left(1-\alpha_{1}\right) \bar{x}+\beta_{1} \bar{x}(1-\bar{x}) e^{-(\bar{x}+\bar{y})} \quad \text { and } \quad \bar{y}=\left(1-\alpha_{2}\right) \bar{y}+\beta_{2} \bar{y}(1-\bar{y}) e^{-(\bar{x}+\bar{y})} \text {. }
$$

Therefore

$$
\bar{x}=0, \quad \alpha_{1}=\beta_{1}(1-\bar{x}) e^{-(\bar{x}+\bar{y})}, \quad \bar{y}=0 \quad \text { and } \quad \alpha_{2}=\beta_{2}(1-\bar{y}) e^{-(\bar{x}+\bar{y})} .
$$

First, set $g(z)=\alpha_{1}-\beta_{1}(1-z) e^{-z}$. Then

$$
g(0)=\alpha_{1}-\beta_{1}, \quad \lim _{z \rightarrow \infty} g(z)=\alpha_{1} \quad \text { and } \quad g^{\prime}(z)=\beta_{1}(2-z) e^{-z}
$$

Therefore $z=2$ is the unique critical point of $g$ and $g(2)$ is the absolute maximum of $g$ on $(0, \infty)$. Now we consider the following two cases. 
(1) If $\alpha_{1} \geq \beta_{1}$, then $g(z) \geq 0$ for all $z>0$ and so $g(z)$ has no positive roots. Similarly, it is easy to show that the function $w(z)=\alpha_{2}-\beta_{2}(1-z) e^{-z}$ has no positive roots provided that $\alpha_{2} \geq \beta_{2}$. Thus System (9) has the unique equilibrium point $(0,0)$.

(2) If $\alpha_{1}<\beta_{1}$, then $g(0)<0$ and since $g^{\prime}(z)>0$ for all $z \in(0,2), g(z)$ has a unique positive root. Since $g(1)=\alpha_{1}>0$, the positive root of $g(z)$ lies in $(0,1)$. So, the equation $\alpha_{1}=\beta_{1}\left(1-x^{*}\right) e^{-x^{*}}$ has a unique solution $x^{*} \in(0,1)$. Similarly, it is easy to show that the equation $\alpha_{2}=\beta_{2}\left(1-y^{*}\right) e^{-y^{*}}$ has a unique solution $y^{*} \in(0,1)$ provided that $\alpha_{2}<\beta_{2}$. Therefore System (9) has the equilibrium points $\left(x^{*}, 0\right)$ and $\left(0, y^{*}\right)$ where $x^{*}$ and $y^{*}$ satisfy $\alpha_{1}=\beta_{1}\left(1-x^{*}\right) e^{-x^{*}}$ and $\alpha_{2}=\beta_{2}\left(1-y^{*}\right) e^{-y^{*}}$, respectively.

Second, assume that $\left(u^{*}, v^{*}\right)$ is a solution of System (10) with $u^{*}>0$ and $v^{*}>0$. It follows from (10) that $u^{*}$ and $v^{*}$ have to be less than one and

$$
e^{u^{*}+v^{*}}=\frac{\beta_{1}}{\alpha_{1}}\left(1-u^{*}\right)=\frac{\beta_{2}}{\alpha_{2}}\left(1-v^{*}\right),
$$

which gives that $v^{*}=\sigma u^{*}-\sigma+1$, where $\sigma=\frac{\alpha_{2} \beta_{1}}{\alpha_{1} \beta_{2}}$. Now set $h(\mu)=\alpha_{1}-\rho_{1}(1-\mu) e^{-\gamma_{1} \mu}$, where $\sigma_{1}=1+\frac{\alpha_{2} \beta_{1}}{\alpha_{1} \beta_{2}}$ and $\rho_{1}=\beta_{1} e^{\sigma_{1}-2}$. Similarly to above, one can easily see that $h$ has no positive roots if $\alpha_{1} \geq \rho_{1}$ and it has a unique positive root which lies in $(0,1)$ whenever $\alpha_{1}<\rho_{1}$. Therefore System (9) has the unique positive equilibrium point $\left(u^{*}, v^{*}\right)$ where $u^{*}$ satisfies $\alpha_{1}=\rho_{1}\left(1-u^{*}\right) e^{-\sigma_{1} u *}$ and $v^{*}=\frac{\alpha_{2} \beta_{1}}{\alpha_{1} \beta_{2}} u^{*}-\frac{\alpha_{2} \beta_{1}}{\alpha_{1} \beta_{2}}+1$.

Recall that $E_{0}, E_{1}$ and $E_{2}$ are called boundary equilibrium points of System (9) and $E_{3}$ is called a Nash equilibrium point of System (9). See [3].

In the following, we deal with the local stability of the equilibrium points of System (9). Now rewrite System (9) as follows:

$$
\left.\begin{array}{l}
x_{n+1}=F\left(x_{n}, y_{n}\right)=\left(1-\alpha_{1}\right) x_{n}+\beta_{1} x_{n}\left(1-x_{n}\right) e^{-\left(x_{n}+y_{n}\right)}, \\
y_{n+1}=G\left(x_{n}, y_{n}\right)=\left(1-\alpha_{2}\right) y_{n}+\beta_{2} y_{n}\left(1-y_{n}\right) e^{-\left(x_{n}+y_{n}\right)}
\end{array}\right\}
$$

where $F(x, y)=\left(1-\alpha_{1}\right) x+\beta_{1} x(1-x) e^{-(x+y)}$ and $G(x, y)=\left(1-\alpha_{2}\right) y+\beta_{2} y(1-y) e^{-(x+y)}$ are continuous functions. Then we obtain

$$
\left.\begin{array}{l}
\frac{\partial F(x, y)}{\partial x}=1-\alpha_{1}+\beta_{1}\left(x^{2}-3 x+1\right) e^{-(x+y)}, \\
\frac{\partial F(x, y)}{\partial y}=-\beta_{1} x(1-x) e^{-(x+y)}, \\
\frac{\partial G(x, y)}{\partial x}=-\beta_{2} y(1-y) e^{-(x+y)}, \\
\frac{\partial G(x, y)}{\partial y}=1-\alpha_{2}+\beta_{2}\left(y^{2}-3 y+1\right) e^{-(x+y)}
\end{array}\right\}
$$

Proposition 2 The equilibrium point $E_{0}$ of System (9) is locally asymptotically stable if $\beta_{i}<\alpha_{i}<2+\beta_{i}$ for $i=1,2$ and it is unstable elsewhere.

Proof The Jacobian matrix of System (9) about the equilibrium point $E_{0}(0,0)$ has the form

$$
J\left(E_{0}\right)=\left[\begin{array}{cc}
1-\alpha_{1}+\beta_{1} & 0 \\
0 & 1-\alpha_{2}+\beta_{2}
\end{array}\right] .
$$

Therefore the eigenvalues of $J\left(E_{0}\right)$ are given by

$$
\lambda_{1}=1-\alpha_{1}+\beta_{1} \quad \text { and } \quad \lambda_{2}=1-\alpha_{2}+\beta_{2} .
$$


It is well known that the equilibrium point $E_{0}$ of System (9) is locally asymptotically stable if both $\left|\lambda_{1}\right|<1$ and $\left|\lambda_{2}\right|<1$ are satisfied if $\beta_{1}<\alpha_{1}<2+\beta_{1}$ and $\beta_{2}<\alpha_{2}<2+\beta_{2}$. The proof is completed.

Proposition 3 The equilibrium points $E_{1}$ and $E_{2}$ of System (9) are saddle points.

Proof The Jacobian matrix of System (9) about the equilibrium point $E_{1}\left(x^{*}, 0\right)$ has the form

$$
J\left(E_{1}\right)=\left[\begin{array}{cc}
1-\alpha_{1}+\beta_{1}\left(x^{* 2}-3 x^{*}+1\right) e^{-x^{*}} & -\beta_{1} x^{*}(1-x) e^{-x^{*}} \\
0 & 1-\alpha_{2}+\beta_{2} e^{-x^{*}}
\end{array}\right] .
$$

Thus $J\left(E_{1}\right)$ has the eigenvalues

$$
\lambda_{1}=1-\alpha_{1}+\beta_{1}\left(x^{* 2}-3 x^{*}+1\right) e^{-x^{*}} \text { and } \quad \lambda_{2}=1-\alpha_{2}+\beta_{2} e^{-x^{*}} .
$$

Note that

$$
\lambda_{1}=1-\alpha_{1}+\beta_{1}\left(x^{* 2}-3 x^{*}+1\right) e^{-x^{*}}<1-\beta_{1} x^{*}\left(2-x^{*}\right) e^{-x^{*}}<1
$$

and

$$
\lambda_{2}=1-\alpha_{2}+\frac{\alpha_{2}}{1-x^{*}}=1+\frac{\alpha_{2} x^{*}}{1-x^{*}}>1
$$

Thus it follows that the equilibrium point $E_{1}\left(x^{*}, 0\right)$ of System (9) is a saddle point. Similarly, one can easily prove that the equilibrium point $E_{2}\left(0, y^{*}\right)$ of System (9) is also a saddle point.

Proposition 4 The Nash equilibrium point $E_{3}$ of System (9) is asymptotically stable if $2<\frac{\alpha_{1} u^{*}\left(2-u^{*}\right)}{1-u^{*}}+\frac{\alpha_{2} v^{*}\left(2-v^{*}\right)}{1-v^{*}}<1+\frac{\alpha_{1} \alpha_{2} u^{*} v^{*}(3-u-v)}{\left(1-u^{*}\right)\left(1-v^{*}\right)}$ and it is unstable elsewhere.

Proof The Jacobian matrix of System (9) about the equilibrium point $E_{3}\left(u^{*}, v^{*}\right)$ is

$$
\begin{aligned}
J\left(E_{3}\right) & =\left[\begin{array}{cc}
1-\alpha_{1}+\frac{\alpha_{1}\left(u^{* 2}-3 u^{*}+1\right)}{1-u^{*}} & -\alpha_{1} u^{*} \\
-\alpha_{2} v^{*} & 1-\alpha_{2}+\frac{\alpha_{2}\left(v^{* 2}-3 v^{*}+1\right)}{1-v^{*}}
\end{array}\right] \\
= & {\left[\begin{array}{cc}
1-\frac{\alpha_{1} u^{*}\left(2-u^{*}\right)}{1-u^{*}} & -\alpha_{1} u^{*} \\
-\alpha_{2} v^{*} & 1-\frac{\alpha_{2} v^{*}\left(2-v^{*}\right)}{1-v^{*}}
\end{array}\right] . }
\end{aligned}
$$

By some simple computations, we obtain that

$$
\begin{aligned}
& \operatorname{Tr}\left(J\left(E_{3}\right)\right)=2-\frac{\alpha_{1} u^{*}\left(2-u^{*}\right)}{1-u^{*}}-\frac{\alpha_{2} v^{*}\left(2-v^{*}\right)}{1-v^{*}}, \\
& \operatorname{Det}\left(J\left(E_{3}\right)\right)=1-\frac{\alpha_{1} u^{*}\left(2-u^{*}\right)}{1-u^{*}}-\frac{\alpha_{2} v^{*}\left(2-v^{*}\right)}{1-v^{*}}+\frac{\alpha_{1} \alpha_{2} u^{*} v^{*}\left(3-u^{*}-v^{*}\right)}{\left(1-u^{*}\right)\left(1-v^{*}\right)} .
\end{aligned}
$$

It is well known that the Nash equilibrium point $E_{3}$ of System (9) is asymptotically stable if $\operatorname{Tr}\left(J\left(E_{3}\right)\right)<0$ and $\operatorname{Det}\left(J\left(E_{3}\right)\right)>0$, i.e., the following condition is satisfied:

$$
2<\frac{\alpha_{1} u^{*}\left(2-u^{*}\right)}{1-u^{*}}+\frac{\alpha_{2} v^{*}\left(2-v^{*}\right)}{1-v^{*}}<1+\frac{\alpha_{1} \alpha_{2} u^{*} v^{*}(3-u-v)}{\left(1-u^{*}\right)\left(1-v^{*}\right)} .
$$

This completes the proof. 


\section{Boundedness and invariant}

In this section we concern ourselves with the boundedness character of the solutions for System (9). Under appropriate conditions, we give some bounded results related to System (9).

Theorem 5 Assume that $\alpha_{i}+\frac{\beta_{i}}{e^{2}}<1, i=1,2$. Then every solution $\left\{\left(x_{n}, y_{n}\right)\right\}_{n=0}^{\infty}$ of System (9), with $x_{0}>0$ and $y_{0}>0$, satisfies that $x_{n}>0$ and $y_{n}>0$ for all $n>0$.

Proof Let $H_{i}(x, y), i=1,2$, be continuous functions defined by

$$
H_{i}(x, y)=1-\alpha_{i}+\beta_{i}(1-x) e^{-(x+y)}, \quad i=1,2 .
$$

Then System (9) can be rewritten in the form

$$
\begin{aligned}
& x_{n+1}=x_{n} H_{1}\left(x_{n}, y_{n}\right), \\
& y_{n+1}=y_{n} H_{2}\left(x_{n}, y_{n}\right) .
\end{aligned}
$$

Now assume that $\left\{\left(x_{n}, y_{n}\right)\right\}_{n=0}^{\infty}$ is a solution of System (9) with positive initial values. Then it suffices to show that $H_{i}(x, y), i=1,2$, are positive for all $x>0, y>0$. Observe that

$$
\frac{\partial H_{i}(x, y)}{\partial x}=\beta_{i}(x-2) e^{-(x+y)} \quad \text { and } \quad \frac{\partial H_{i}(x, y)}{\partial y}=-\beta_{i}(1-x) e^{-(x+y)}, \quad i=1,2 .
$$

Therefore $H_{1}$ and $H_{2}$ have no positive critical points. Let $a$ and $b$ be arbitrary positive numbers and consider the domain

$$
D=\{(x, y): 0 \leq x \leq a, 0 \leq y \leq b\} .
$$

Then for $i=1,2$, we see that

$$
\begin{aligned}
& H_{i}(0, y)=1-\alpha_{i}+\beta_{i} e^{-y}, \quad 0 \leq y \leq b, \\
& H_{i}(x, 0)=1-\alpha_{i}+\beta_{i}(1-x) e^{-x}, \quad 0 \leq x \leq a, \\
& H_{i}(x, b)=1-\alpha_{i}+\beta_{i}(1-x) e^{-(x+b)}, \quad 0 \leq x \leq a, \\
& H_{i}(a, y)=1-\alpha_{i}+\beta_{i}(1-a) e^{-(a+y)}, \quad 0 \leq y \leq b .
\end{aligned}
$$

Using elementary differential calculus, we obtain that the absolute minimum of each one of the above functions is $1-\alpha_{i}-\frac{\beta_{i}}{e^{2}}$. Therefore $H_{i}(x, y) \geq 1-\alpha_{i}-\frac{\beta_{i}}{e^{2}}>0$ for all $(x, y) \in D$. Since $a$ and $b$ are arbitrary positive numbers, we can conclude that $H_{i}(x, y)>0$ for $i=1,2$ and for all $(x, y) \in(0, \infty)^{2}$.

Theorem 6 Let $\left\{\left(x_{n}, y_{n}\right)\right\}_{n=0}^{\infty}$ be a solution of System (9) with $\left(x_{n_{0}}, y_{n_{0}}\right) \in(0,1]^{2}$ for some $n_{0} \geq 0$ and assume, for $i=1,2$, that one of the following statements is true:

(i) $\beta_{i} \leq e\left(1-\alpha_{i}\right)$.

(ii) $e\left(1-\alpha_{i}\right)<\beta_{i} \leq e$.

(iii) $\left(\sqrt{\beta_{i}}-1\right)^{2} \leq \alpha_{i}$.

Then $\left(x_{n}, y_{n}\right) \in(0,1]^{2}$ for all $n \geq n_{0}$. 
Proof Let $n_{0} \geq 0$ be such that $x_{n_{0}} \in(0,1]$. It follows from System (9) that

$$
\begin{aligned}
& x_{n_{0}+1} \leq\left(1-\alpha_{1}\right) x_{n_{0}}+\beta_{1}\left(1-x_{n_{0}}\right) x_{n_{0}} e^{-x_{n_{0}}}, \\
& x_{n_{0}+1} \leq\left(1-\alpha_{1}\right) x_{n_{0}}+\beta_{1}\left(1-x_{n_{0}}\right) e^{-1}=\left(1-\alpha_{1}-\frac{\beta_{1}}{e}\right) x_{n_{0}}+\frac{\beta_{1}}{e}
\end{aligned}
$$

and

$$
x_{n_{0}+1} \leq\left(1-\alpha_{1}\right) x_{n_{0}}+\beta_{1}\left(1-x_{n_{0}}\right) x_{n_{0}} .
$$

Set $w(x)=\left(1-\alpha_{1}\right) x+\beta_{1}(1-x) x e^{-x}$ for $x \leq 1$. Then it follows from (13) that $x_{n_{0}+1} \leq w\left(x_{n_{0}}\right)$. Also, we obtain that

$$
w^{\prime}(x)=\left(1-\alpha_{1}\right)+\beta_{1}\left(x^{2}-3 x+1\right) e^{-x}
$$

and

$$
\begin{aligned}
w^{\prime \prime}(x) & =\beta_{1}\left(-x^{2}+5 x-4\right) e^{-x}=-\beta_{1}(x-1)(x-4) e^{-x} \\
& \leq 0 \quad \text { for all } x \in(0,1] .
\end{aligned}
$$

Then $w^{\prime}(x) \geq w^{\prime}(1)=1-\alpha_{1}-\frac{\beta_{1}}{e}$. If (i) holds, then $w^{\prime}(1) \geq 0$ and hence $w(x)$ is increasing on $(0,1]$. Therefore $x_{n_{0}+1} \leq w(1)<1$. If (ii) holds, then (14) yields $x_{n_{0}+1} \leq \frac{\beta_{1}}{e}<1$.

Now suppose that (iii) holds. In this case, it follows from (15) that $x_{n_{0}+1} \leq p\left(x_{n_{0}}\right)$, where $p(x)=\left(1-\alpha_{1}\right) x+\beta_{1} x(1-x)$ for all $x \in(0,1]$. It is not difficult to see that $p\left(x_{*}\right)$ is the absolute maximum of $p(x)$ on $(0,1]$ where $x_{*}=\frac{1-\alpha_{1}+\beta_{1}}{2 \beta_{1}}$. According to (iii) and since $p\left(x_{*}\right)=\frac{\left(1-\alpha_{1}+\beta_{1}\right)^{2}}{4 \beta_{1}} \leq 1, x_{n_{0}+1} \leq p\left(x_{*}\right) \leq 1$. That is, in all cases we obtain that whenever $x_{n_{0}} \leq 1$ yields $x_{n_{0}+1} \leq 1$. So it is easy to prove by induction that $x_{n} \in(0,1]$ for all $n \geq 1$. The proof of $y_{n}$ is similar and so will be omitted. This completes the proof.

Theorem 7 For every solution $\left\{\left(x_{n}, y_{n}\right)\right\}_{n=0}^{\infty}$ of System (9), the following statements hold:

(i) $x_{n} \leq x_{n_{0}}\left(1-\alpha_{1}\right)^{n-n_{0}}+\frac{\beta_{1}}{e \alpha_{1}}\left(1-\left(1-\alpha_{1}\right)^{n-n_{0}}\right), n \geq n_{0} \geq 0$.

(ii) $y_{n} \leq y_{n_{0}}\left(1-\alpha_{2}\right)^{n-n_{0}}+\frac{\beta_{2}}{e \alpha_{2}}\left(1-\left(1-\alpha_{2}\right)^{n-n_{0}}\right), n \geq n_{0} \geq 0$.

Proof We obtain, for $n_{0} \geq 0$, from System (9) that

$$
\begin{aligned}
x_{n+1} & \leq\left(1-\alpha_{1}\right) x_{n}+\beta_{1} x_{n} e^{-x_{n}} \\
& \leq\left(1-\alpha_{1}\right) x_{n}+\frac{\beta_{1}}{e} \quad \text { for all } n \geq n_{0} .
\end{aligned}
$$

Then it follows by Theorem 5 and Theorem 6 that Case (i) is true. The proof of Case (ii) is similar and will be omitted.

The following corollaries are coming immediately from Theorem 7.

Corollary 8 Assume that $\left\{\left(x_{n}, y_{n}\right)\right\}_{n=0}^{\infty}$ is a positive solution of System (9) with $\left(x_{n_{0}}, y_{n_{0}}\right) \in$ $\left(0, \frac{\beta_{1}}{\alpha_{1} e}\right] \times\left(0, \frac{\beta_{2}}{\alpha_{2} e}\right]$ for some $n_{0} \geq 0$. Then $\left(x_{n}, y_{n}\right) \in\left(0, \frac{\beta_{1}}{\alpha_{1} e}\right] \times\left(0, \frac{\beta_{2}}{\alpha_{2} e}\right]$ for all $n \geq n_{0}$. 
Corollary 9 Every positive solution $\left\{\left(x_{n}, y_{n}\right)\right\}_{n=0}^{\infty}$ of System (9) is bounded. Moreover,

$$
\limsup _{n \rightarrow \infty} x_{n} \leq \frac{\beta_{1}}{\alpha_{1} e}
$$

and

$$
\limsup _{n \rightarrow \infty} y_{n} \leq \frac{\beta_{2}}{\alpha_{2} e} .
$$

Theorem 10 Assume that $\left\{\left(x_{n}, y_{n}\right)\right\}_{n=0}^{\infty}$ is a positive solution of System (9) and assume, for $i=1,2$, that one of the following conditions is true:

(i) $\beta_{i}<\alpha_{i} e$.

(ii) $2+v_{i}^{2}+2 e^{v_{i}}-4 v_{i}-v_{i} e^{v_{i}}>0,1-\alpha_{i}+\beta_{i} e^{-v_{i}}\left[1-v_{i}\left(2 e^{-v_{i}}+1\right)+v_{i}^{2} e^{-v_{i}}\right]$ and $(1-\alpha) v_{i}+\beta_{i} v_{i} e^{-v_{i}}-\beta v_{i}^{2} e^{-2 v_{i}}<1$, where $v_{i}=\frac{\beta_{i}}{\alpha_{i} e}$.

Then there exists $n_{0} \geq 0$ such that $\left(x_{n}, y_{n}\right) \in(0,1]^{2}$ for all $n \geq n_{0}$.

Proof The proof of the theorem, when (i) holds, follows by Corollary 9. Now consider that (ii) is true. Then it follows from Corollary 9 that for every constant $\varepsilon_{1}>0$, there exists $n_{0} \geq 0$ such that $x_{n} \leq \frac{\beta_{1}}{\alpha_{1} e}+\varepsilon_{1}=\gamma_{1}, n \geq n_{0}$. Set $\delta_{1}=e^{-\gamma_{1}}$. Since $\delta_{1} \rightarrow e^{-\nu_{1}}$ when $\varepsilon_{1} \rightarrow 0$ and the inequalities in (ii) hold, depending on the continuity in $v_{1}$ of the left-hand side of each inequality in (ii), one can choose $\varepsilon_{1}$ so small that

$$
\begin{aligned}
& 2+\gamma_{1}^{2}+\frac{2-\gamma_{1}}{\delta_{1}}-4 \gamma_{1} \geq 0, \\
& 1-\alpha_{1}+\beta_{1} \delta_{1}\left[1-\left(2 \delta_{1}+1\right) \gamma_{1}+\delta_{1} \gamma_{1}^{2}\right] \geq 0
\end{aligned}
$$

and

$$
\left(1-\alpha_{1}\right) \gamma_{1}+\beta_{1} \gamma_{1} \delta_{1}-\beta_{1} \gamma_{1}^{2} \delta_{1}^{2} \leq 1
$$

Now we obtain from System (9) that

$$
\begin{aligned}
x_{n+1} & =\left(1-\alpha_{1}\right) x_{n}+\beta_{1} x_{n} e^{-\left(x_{n}+y_{n}\right)}-\beta_{1} x_{n}^{2} e^{-\left(x_{n}+y_{n}\right)} \\
& \leq\left(1-\alpha_{1}\right) x_{n}+\beta_{1} x_{n} e^{-x_{n}}-\beta_{1} \delta_{1} x_{n}^{2} e^{-x_{n}} \\
& =K\left(x_{n}\right), \quad n \geq n_{0},
\end{aligned}
$$

where $K(x)=\left(1-\alpha_{1}\right) x+\beta_{1} e^{-x}\left(x-\delta_{1} x^{2}\right), x \leq \gamma_{1}$ and then

$$
K^{\prime}(x)=1-\alpha_{1}+\beta_{1} e^{-x}\left[\delta_{1} x^{2}-\left(2 \delta_{1}+1\right) x+1\right]
$$

and

$$
K^{\prime \prime}(x)=-\beta_{1} e^{-x}\left[\delta_{1} x^{2}-\left(4 \delta_{1}+1\right) x+2\left(\delta_{1}+1\right)\right] .
$$

On the other hand, the equation

$$
\delta_{1} x^{2}-\left(4 \delta_{1}+1\right) x+2\left(\delta_{1}+1\right)=0
$$


has the positive roots

$$
x_{1}=\frac{4 \delta_{1}+1+\sqrt{8 \delta_{1}^{2}+1}}{2 \delta_{1}} \text { and } x_{2}=\frac{4 \delta_{1}+1-\sqrt{8 \delta_{1}^{2}+1}}{2 \delta_{1}} \text {. }
$$

Observe that $x_{2}=2+\frac{1}{2 \delta_{1}}-\sqrt{2+\frac{1}{4 \delta_{1}^{2}}} \geq \gamma_{1}$ if and only if $\left(2+\frac{1}{2 \delta_{1}}-\gamma_{1}\right)^{2} \geq 2+\frac{1}{4 \delta_{1}^{2}}$ which holds by (16). Therefore $x_{1} \geq x_{2} \geq \gamma_{1}$. Consequently, $K^{\prime \prime}(x)<0$ for all $x \leq \gamma_{1}$, which yields by (17) that $K^{\prime}(x)>K^{\prime}\left(\gamma_{1}\right) \geq 0$. Using the increasing property of $K(x)$ on $\left(0, \gamma_{1}\right)$ and inequality (18), we see that $K(x) \leq K\left(\gamma_{1}\right) \leq 1$. Since $x_{n} \leq \gamma_{1}$, it follows that

$$
x_{n+1} \leq K\left(x_{n}\right) \leq K\left(\gamma_{1}\right) \leq 1 \text { for all } n \geq n_{0} .
$$

Similarly, one can easily prove that $y_{n} \in(0,1]$. This completes the proof.

Theorem 11 Assume that $\left\{\left(x_{n}, y_{n}\right)\right\}_{n=0}^{\infty}$ is a positive solution of System (9). If either

$$
\left(1-\alpha_{i}+\beta_{i}\right)^{2}<4 \beta_{i} e^{-2 v_{i}}
$$

or

$$
\frac{\beta_{i} e^{v_{i}}}{4}+\beta_{i}\left(1-\alpha_{i}\right)<1
$$

where $v_{i}=\frac{\beta_{i}}{\alpha_{i} e}$ for $i=1,2$, then there exists $n_{0} \geq 0$ such that $\left(x_{n}, y_{n}\right) \in(0,1]^{2}$ for all $n \geq n_{0}$.

Proof Assume that $\gamma_{1}, \delta_{1}$ and the function $K\left(x_{n}\right)$ are defined as in the previous proof. Then

$$
K\left(x_{n}\right)=\left(1-\alpha_{1}+\beta_{1}\right) x_{n}-\beta_{1} x_{n}^{2} \delta_{1}^{2}=\bar{K}\left(x_{n}\right),
$$

where $\bar{K}(x)=\left(1-\alpha_{1}+\beta_{1}\right) x-\beta_{1} x^{2} \delta_{1}^{2}, x \leq \gamma_{1}$. Thus

$$
\bar{K}^{\prime}(x)=1-\alpha_{1}+\beta_{1}-2 \beta_{1} x \delta_{1}^{2} .
$$

Hence, $\bar{K}(x)$ attains its maximum value at $x=\frac{1-\alpha_{1}+\beta_{1}}{2 \beta_{1} \delta_{1}^{2}}$, that is,

$$
\bar{K}(x) \leq \bar{K}\left(\frac{1-{ }_{1} \alpha_{1}+\beta_{1}}{2 \beta_{1} \delta_{1}^{2}}\right)=\frac{\left(1-\alpha_{1}+\beta_{1}\right)^{2}}{4 \beta_{1} \delta_{1}^{2}}
$$

Also,

$$
\begin{aligned}
K\left(x_{n}\right) & =-\beta_{1} \delta_{1} e^{-x_{n}}\left(x_{n}-\frac{1}{2 \delta_{1}}\right)^{2}+\frac{\beta_{1} e^{-x_{n}}}{4 \delta_{1}}+\beta_{1}\left(1-\alpha_{1}\right) \\
& <\frac{\beta_{1}}{4 \delta_{1}}+\beta_{1}\left(1-\alpha_{1}\right), \quad n \geq n_{0} .
\end{aligned}
$$

Similarly to the proof of Theorem 10 , we can choose $\varepsilon_{1}$ so small that our assumptions imply

$$
\frac{\left(1-\alpha_{1}+\beta_{1}\right)^{2}}{2 \beta_{1} \delta_{1}^{2}} \leq 1 \text { and } \frac{\beta_{1}}{4 \delta_{1}}+\beta_{1}\left(1-\alpha_{1}\right) \leq 1
$$


Therefore we have either

$$
x_{n+1} \leq \bar{K}\left(x_{n}\right) \leq \frac{\left(1-\alpha_{1}+\beta_{1}\right)^{2}}{2 \beta_{1} \delta_{1}^{2}} \leq 1, \quad n \geq n_{0}
$$

or

$$
x_{n+1} \leq K\left(x_{n}\right) \leq \frac{\beta_{1}}{4 \delta_{1}}+\beta_{1}\left(1-\alpha_{1}\right) \leq 1, \quad n \geq n_{0},
$$

which is our desired conclusion for $x_{n}$. Similarly, one can accomplish the same conclusion for $y_{n}$. So, the proof is complete.

\section{Global stability analysis}

In this section we are interested in driving conditions under which the equilibrium points of System (9) are attractors of the solutions for System (9).

In the following theorem, we investigate the global attractivity of the equilibrium point $(0,0)$ of System (9).

Theorem 12 Assume that $\alpha_{i} \geq \beta_{i}, i=1,2$. Then $(0,0)$ is a global attractor of all positive solutions of System (9).

Proof Let $\left\{\left(x_{n}, y_{n}\right)\right\}_{n=0}^{\infty}$ be a solution of System (1). It follows from System (1) that

$$
x_{n+1}=\left(1-\alpha_{1}\right) x_{n}+\beta_{1} x_{n}\left(1-x_{n}\right) e^{-\left(x_{n}+y_{n}\right)}<\left(1-\alpha_{1}+\beta_{1}\right) x_{n}<x_{n}
$$

and

$$
y_{n+1}=\left(1-\alpha_{2}\right) y_{n}+\beta_{2} y_{n}\left(1-y_{n}\right) e^{-\left(x_{n}+y_{n}\right)}<\left(1-\alpha_{2}+\beta_{2}\right) y_{n}<y_{n} .
$$

Then there exist $x \geq 0$ and $y \geq 0$ such that $\lim _{n \rightarrow \infty} x_{n}=x$ and $\lim _{n \rightarrow \infty} y_{n}=y$. Since the only possible values of $(x, y)$ in the present case are $(0,0), \lim _{n \rightarrow \infty} x_{n}=0$ and $\lim _{n \rightarrow \infty} y_{n}=0$. This completes the proof.

In the following theorems, we investigate the global attractivity of the positive equilibrium point $(\bar{x} ; \bar{y})$ of System (9), where $\bar{x}$ and $\bar{y}$ are given by $\alpha_{1}=\beta_{1}(1-\bar{x}) e^{-(\bar{x}+\bar{y})}$ and $\alpha_{2}=\beta_{2}(1-\bar{y}) e^{-(\bar{x}+\bar{y})}$, respectively.

Theorem 13 Assume that $\alpha_{i}+\beta_{i} e^{-2}<1, i=1,2$. Then the unique positive equilibrium point $(\bar{x} ; \bar{y})$ of System (9) is a global attractor of all positive solutions of System (9).

Proof Let $\left\{\left(x_{n}, y_{n}\right)\right\}_{n=0}^{\infty}$ be a solution of System (9) and let $x_{n} \leq \bar{x}$ (the case whenever $x_{n} \geq \bar{x}$ is similar and it will be left to the reader). Since $x_{n} \leq \bar{x}$, then $h\left(x_{n}\right) \leq 0$, where $h\left(x_{n}\right)=$ $\alpha_{1}-\beta_{1}\left(1-x_{n}\right) e^{-\left(x_{n}+y_{n}\right)}$. Thus $\alpha_{1} \leq \beta_{1}\left(1-x_{n}\right) e^{-\left(x_{n}+y_{n}\right)}$. Therefore we obtain from System (9) that

$$
\begin{aligned}
x_{n+1} & =\left(1-\alpha_{1}\right) x_{n}+\beta_{1} x_{n}\left(1-x_{n}\right) e^{-\left(x_{n}+y_{n}\right)} \\
& \geq\left(1-\alpha_{1}\right) x_{n}+\alpha_{1} x_{n}=x_{n} .
\end{aligned}
$$


Then the sequence $\left\{x_{n}\right\}_{n=0}^{\infty}$ is increasing and since it was shown that it is bounded above, then it converges to the unique positive equilibrium point $\bar{x}$. Similarly, assume that $y_{n} \leq \bar{y}$ (the case whenever $y_{n} \geq \bar{y}$ is similar and it will be left to the reader). Since $y_{n} \leq \bar{y}$, then $g\left(y_{n}\right) \leq 0$, where $g\left(y_{n}\right)=\alpha_{2}-\beta_{2}\left(1-y_{n}\right) e^{-\left(x_{n}+y_{n}\right)}$. Thus $\alpha_{2} \leq \beta_{2}\left(1-y_{n}\right) e^{-\left(x_{n}+y_{n}\right)}$. Therefore we obtain from System (9) that

$$
\begin{aligned}
y_{n+1} & =\left(1-\alpha_{2}\right) y_{n}+\beta_{2} y_{n}\left(1-y_{n}\right) e^{-\left(x_{n}+y_{n}\right)} \\
& \geq\left(1-\alpha_{2}\right) y_{n}+\alpha_{2} y_{n}=y_{n} .
\end{aligned}
$$

Then, again, the sequence $\left\{y_{n}\right\}_{n=0}^{\infty}$ is increasing, and since it was shown that it is bounded above, then it converges to the unique positive equilibrium point $\bar{y}$. Thus $\left\{\left(x_{n}, y_{n}\right)\right\}_{n=0}^{\infty}$ converges to $(\bar{x} ; \bar{y})$.

Theorem 14 Consider $\alpha_{1}=\alpha_{2}=\alpha$ and $\beta_{1}=\beta_{2}=\beta$ and assume that $\beta(\alpha e-\beta) \geq \alpha^{2} e^{3}$. Then the unique positive equilibrium point $(\bar{x} ; \bar{y})$ of System (9) is a global attractor of all positive solutions of System (9).

Proof Let $\left\{\left(x_{n}, y_{n}\right)\right\}_{n=0}^{\infty}$ be a solution of System (9). It follows from System (9) that

$$
\begin{aligned}
x_{n+1} & =(1-\alpha) x_{n}+\beta x_{n}\left(1-x_{n}\right) e^{-\left(x_{n}+y_{n}\right)} \\
& \geq(1-\alpha) x_{n}+\beta x_{n}\left(1-x_{n}\right) e^{-2} .
\end{aligned}
$$

Thus we see from Corollary 9 that

$$
x_{n+1} \geq\left[1-\alpha+\beta\left(1-\frac{\beta}{\alpha e}\right) e^{-2}\right] x_{n} \geq x_{n}
$$

Then the sequence $\left\{x_{n}\right\}_{n=0}^{\infty}$ is increasing and since it is bounded, then it converges to the unique positive equilibrium point $\bar{x}$. Similarly, it is easy to show that the sequence $\left\{y_{n}\right\}_{n=0}^{\infty}$ is also convergent to the unique positive equilibrium point $\bar{y}=\bar{x}$ : Therefore $\left\{\left(x_{n}, y_{n}\right)\right\}_{n=0}^{\infty}$ converges to $(\bar{x}, \bar{y})$ and then the proof is complete.

Theorem 15 Consider $\alpha_{1}=\alpha_{2}=\alpha$ and $\beta_{1}=\beta_{2}=\beta$ and assume that one of the following conditions holds:

(I) $5 \beta \leq 4 e^{2}(1-\alpha)$.

(II) $\alpha+\beta<1$.

Then the unique positive equilibrium point $(\bar{x}, \bar{x})$ of System (9) is a global attractor of all positive solutions of System (9).

Proof Rewrite System (9) as follows:

$$
\left.\begin{array}{l}
x_{n+1}=F\left(x_{n}, y_{n}\right)=(1-\alpha) x_{n}+\beta\left(1-x_{n}\right) x_{n} e^{-\left(x_{n}+y_{n}\right)} \\
y_{n+1}=G\left(x_{n}, y_{n}\right)=(1-\alpha) y_{n}+\beta\left(1-y_{n}\right) y_{n} e^{-\left(x_{n}+y_{n}\right)}
\end{array}\right\} \quad n=0,1, \ldots
$$


where $F(x, y)=(1-\alpha) x+\beta(1-x) x e^{-(x+y)}$ and $G(x, y)=(1-\alpha) y+\beta(1-y) y e^{-(x+y)}$ are continuous functions. Now consider the system

$$
\left.\begin{array}{l}
m_{1}=F\left(m_{1}, M_{2}\right), \quad M_{1}=F\left(M_{1}, m_{2}\right), \\
m_{2}=G\left(M_{1}, m_{2}\right), \quad M_{2}=F\left(m_{1}, M_{2}\right) .
\end{array}\right\}
$$

Then

$$
\begin{aligned}
& m_{1}=(1-\alpha) m_{1}+\beta m_{1}\left(1-m_{1}\right) e^{-\left(m_{1}+M_{2}\right)}, \\
& M_{1}=(1-\alpha) M_{1}+\beta M_{1}\left(1-M_{1}\right) e^{-\left(M_{1}+m_{2}\right)}, \\
& m_{2}=(1-\alpha) m_{2}+\beta m_{2}\left(1-m_{2}\right) e^{-\left(m_{2}+M_{1}\right)}, \\
& M_{2}=(1-\alpha) M_{2}+\beta M_{2}\left(1-M_{2}\right) e^{-\left(M_{2}+m_{1}\right)} .
\end{aligned}
$$

Thus either $m_{1}=M_{2}=m_{2}=M_{2}$ or

$$
\begin{aligned}
& \alpha=\beta\left(1-m_{1}\right) e^{-\left(m_{1}+M_{2}\right)}, \\
& \alpha=\beta\left(1-M_{1}\right) e^{-\left(M_{1}+m_{2}\right)}, \\
& \alpha=\beta\left(1-m_{2}\right) e^{-\left(m_{2}+M_{1}\right)}, \\
& \alpha=\beta\left(1-M_{2}\right) e^{-\left(M_{2}+m_{1}\right)} .
\end{aligned}
$$

Then $m_{1}=M_{2}, m_{2}=M_{2}$ and $\left(1-m_{1}\right) e^{-2 m_{1}}=\left(1-M_{1}\right) e^{-2 M_{1}}=\left(1-m_{2}\right) e^{-2 m_{2}}=\left(1-M_{2}\right) e^{-2 M_{2}}$. Now since $\left(1-m_{1}\right) e^{-2 m_{1}}=\left(1-M_{1}\right) e^{-2 M_{1}}$, then $e^{2\left(M_{1}-m_{1}\right)}=\frac{1-M_{1}}{1-m_{1}}$, that is,

$$
2\left(M_{1}-m_{1}\right)=\log \left(1-M_{1}\right)-\log \left(1-m_{1}\right) .
$$

We claim that $M_{1}=m_{1}$; otherwise, for the sake of contradiction, assume that $M_{1}>m_{1}$ (the case where $M_{1} \leq m_{1}$ is similar and it will be left to the reader). Then $\log \left(1-M_{1}\right)-\log (1-$ $\left.m_{1}\right)>0 \Rightarrow \log \left(1-M_{1}\right)>\log \left(1-m_{1}\right) \Rightarrow M_{1}<m_{1}$, which is a contradiction.

Now it is easy to see that

$$
\left.\begin{array}{l}
\frac{\partial F(x, y)}{\partial x}=1-\alpha+\beta\left(x^{2}-3 x+1\right) e^{-(x+y)}, \\
\frac{\partial F(x, y)}{\partial y}=-x \beta(1-x) e^{-(x+y)}, \\
\frac{\partial G(x, y)}{\partial x}=-\beta y(1-y) e^{-(x+y)}, \\
\frac{\partial G(x, y)}{\partial y}=1-\alpha+\beta\left(y^{2}-3 y+1\right) e^{-(x+y)}
\end{array}\right\}
$$

Thus

$$
\begin{aligned}
\frac{\partial F(x, y)}{\partial x} & =1-\alpha+\beta\left(x^{2}-3 x+1\right) e^{-(x+y)} \\
& \geq 1-\alpha+\beta\left(x^{2}-3 x+1\right) e^{-2} \\
& =\beta e^{-2} x^{2}-3 \beta e^{-2} x+\beta e^{-2}+1-\alpha .
\end{aligned}
$$

Now, there are two cases to consider: 
Case 1: Suppose that $5 \beta \leq 4 e^{2}(1-\alpha)$. Therefore the function $w(x)=\beta e^{-2} x^{2}-3 \beta e^{-2} x+$ $\beta e^{-2}+1-\alpha$ has no real roots. Thus $\frac{\partial F(x, y)}{\partial x} \geq 0$. Similarly, it is easy to prove that $\frac{\partial G(x, y)}{\partial x} \geq 0$. Then it follows by Theorem A that the equilibrium point $(\bar{x}, \bar{y})=(\bar{x}, \bar{x})$ of System (9) is a global attractor of all positive solutions of System (9).

Case 2: Suppose that $\alpha+2 \beta<1$. Since $0 \leq x \leq 1,3 \geq 3-x \geq x(3-x)=3 x-x^{2}$, or $2 \geq$ $3 x-x^{2}-1$, and since $\alpha+2 \beta<1$, then $1-\alpha>2 \beta>2 \beta e^{-2} \geq 2 \beta e^{-(x+y)} \geq \beta\left(3 x-x^{2}-1\right) e^{-(x+y)}$. Thus $\frac{\partial F(x, y)}{\partial x} \geq 0$. Similarly, it is easy to prove that $\frac{\partial G(x, y)}{\partial x} \geq 0$. Then it follows again by Theorem A that the equilibrium point $(\bar{x}, \bar{y})=(\bar{x}, \bar{x})$ of System (9) is a global attractor of all positive solutions of System (9). Thus the proof is now completed.

\section{Competing interests}

The author declares that they have no competing interests.

\section{Acknowledgements}

This paper was funded by the Deanship of Scientific Research (DSR), King Abdulaziz University, Jeddah, Saudi Arabia under grant No. (662-009-D1433). The author, therefore, acknowledge with thanks DSR technical and financial support. Last, but not least, sincere appreciations are dedicated to all our colleagues in the Faculty of Science, Rabigh branch for their nice wishes.

Received: 5 January 2013 Accepted: 23 May 2013 Published: 17 June 2013

\section{References}

1. Bischi, Gl, Naimzada, A: Global analysis of a dynamic duopoly game with bounded rationality. In: Advances in Dynamic Games and Application, vol. 5, chapter 20. Birkhäuser, Boston (1999)

2. Cournot, A: Researches Into the Mathematical Principles of the Theory of Wealth. Irwin Paper Back Classics in Economics (1963). Engl. trans., chapter VII

3. Agiza, HN, Elsadany, AA: Nonlinear dynamics in the Cournot duopoly game with heterogeneous players. Physica A 320, 512-524 (2003)

4. Agiza, HN, Hegazi, AS, Elsadany, AA: The dynamics of Bowley's model with bounded rationality. Chaos Solitons Fractals 9, 1705-1717 (2001)

5. Agiza, HN, Hegazi, AS, Elsadany, AA: Complex dynamics and synchronization of a duopoly game with bounded rationality. Math. Comput. Simul. 58, 133-146 (2002)

6. Ahmed, E, Agiza, HN, Hassan, SZ: On modifications of Puu's dynamical duopoly. Chaos Solitons Fractals 7, 1025-1028 (2000)

7. Bischi, Gl, Stefanini, L, Gardini, L: Synchronization intermittency and critical curves in a duopoly game. Math. Comput. Simul. 44, 559-585 (1998)

8. Du, J, Huang, T: New results on stable region of Nash equilibrium point of output game model. Appl. Math. Comput. 192, 12-19 (2007)

9. Kopel, M: Simple and complex adjustment dynamics in Cournot duopoly models. Chaos Solitons Fractals 12, 2031-2048 (1996)

10. Yao, HN, Xu, F: Complex dynamics analysis for a duopoly advertising model with nonlinear cost. Appl. Math. Comput. $180,134-145(2006)$

11. Puu, T: Attractors, Bifurcations and Chaos: Nonlinear Phenomena in Economics. Springer, Berlin (2000)

12. El-Metwally, H: Global behavior of an economic model. Chaos Solitons Fractals 33, 994-1005 (2007)

13. Kulenovic, MRS, Nurkanovic, M: Global behavior of a two-dimensional competitive system of difference equations with stocking. Math. Comput. Model. 55, 1998-2011 (2012)

14. Zhang, J, Da, Q, Wang, Y: Analysis of nonlinear duopoly game with heterogeneous players. Econ. Model. 24, 138-148 (2007)

15. Puu, T: Dynamics of Stackelberg duopoly. In: Puu, T, Panchuk, A (eds.) Advances in Nonlinear Economic Dynamics. Nova Science Publishers, New York (2010) 